\title{
Conventional Big Bang Theories and Tired Light Theories
}

\author{
Sumitkumar Bajpeyee \\ Research Scholar M.Phil BRA University Muzaffarpur Bihar \\ M.Sc (Maths) M.S College Motihari
}

\begin{abstract}
The big bang or hot expanding model of cosmology finds strong support from the recession of the galaxies, that is, when we look out in the night sky, distant objects appear to be flying away from us. And also the cosmic microwave background - the fact that we are bathed in primordial light that bears evidence from and earlier, hotter, and denser period in the universe. Tired light is a class of hypothetical redshift mechanisms that was proposed as an alternative explanation for the redshift-distance relationship. ... Zwicky himself acknowledged that any sort of scattering of light would blur the images of distant objects more than what is seen.
\end{abstract}

Keywords: Redshifts, Luminosities, Axions, Photons etc.

\section{Introduction}

Bing Bang model widely held theory of the evolution of the universe. Its essential feature is the emergence of the universe from a state of extremely high temperature and density - the so-called big bang that occurred 13.8 billion years ago. Although this type of universe was proposed by Russian mathematician Aleksandr Friedmann and Belgian astronomer Georges Lemaitre in the 1920s, the modern version was developed by Russian-born American physicist George Gamow and colleagues in the 1940s. The big-bang model is based on two assumptions. The first is that Albert Einstein's general theory of relativity correctly describes the gravitational interaction of all matter. The second assumption, called the cosmological principle, states that an observer's view of the universe depends neither on the direction in which he looks nor on his location. This principle applies only to the large-scale properties of the universe, but it does imply that the universe has no edge, so that the big-bang origin occurred not at a particular point in space but rather throughout space at the same time. These two assumptions make it possible to calculate the history of the cosmos after a certain epoch called the Planck time. Scientists have yet to determine what prevailed before Planck timeAccording to the big-bang model, the universe expanded rapidly from a highly compressed primordial state, which resulted in a significant decrease in density and temperature. Soon afterward, the dominance of matter over antimatter (as observed today) may have been established by processes that also predict proton decay. During this stage many types of elementary particles may have been present. After a few seconds, the universe cooled enough to allow the formation of certain nuclei. The theory predicts that definite amounts of hydrogen, helium, and lithium were produced. Their abundances agree with what is observed today. About one million years later the universe was sufficiently cool for atoms to form. The radiation that also filled the universe was then free to travel through space. This remnant of the early universe is the cosmic microwave background radiation - the - three degree" (actually $2.728 \mathrm{~K}$ ) background radiationdiscovered in 1965 by American physicists Arno A. Penzias and Robert W. Wilson. In addition to accounting for the presence of ordinary matter and radiation, the model predicts that the present universe should also be filled with neutrinos, fundamental particles with no mass or electric charge. The possibility exists that other relics from the early universe may eventually be discovered.

The more distant a galaxy, the more its light is redshifted. This effect, first observed by Edwin Hubble, is one of the main points of evidence for cosmic expansion. But this redshift effect is often misunderstood. After all, if light from a distant galaxy is red shifted, and the energy of a photon depends upon its wavelength, doesn't that mean the photon is spontaneously losing energy. While the energy of a photon does depend upon its wavelength, and its energy is conserved, that only tells part of the story. While energy is conserved in a particular reference frame, the amount of energy we observe can vary depending upon our frame of reference. Imagine, for example, that we are riding in a train, and we place a cup of coffee on a table. From our vantage point the coffee is just sitting in place. From our perspective it has no kinetic energy, and it will continue to sit on the table as long as the train continues to move at a constant speed. From a pedestrian standing outside the train the coffee mug (and the train) are moving along at some speed. It has some kinetic energy because of its motion. From both vantage points the kinetic energy of the coffee cup is constant, but the amount of energy seen from each viewpoint is different. The same is true with light. Of course the redshift from cosmic expansion is different from that of relative motion. With cosmic redshift space expands between the time light is emitted and the time it is absorbed, so it seems as if the light spontaneously redshifts as it travels. That's really going on is that spacetime is warped, giving the illusion of spontaneous redshift, just as the warping of space can cause the path of light to bend in space even though light always follows the shortest path through spacetime. The energy of a photon is still conserved along its path, even though the galaxy that emits the photon and our observation of the photon will perceive different energies.This is why the cosmic redshift model is very different from the so-called tired light model, which assumes that light spontaneously loses energy rather than the universe is expanding. Not only does tired light violate conservation of energy, it makes very different predictions about how the universe would look. When we 


\section{International Journal of Science and Research (IJSR) \\ ISSN (Online): 2319-7064}

Index Copernicus Value (2015): 78.96 | Impact Factor (2015): 6.391

compare it to observation, the tired light model fails. So even though the light from distant galaxies are redshifted, the light's energy is still conserved.

\section{Literature Survey}

The Big Bang theory developed from observations of the structure of the universe and from theoretical considerations . In 1912 Vestoslipher measured the first Doppler shift of a -spiral nebula" and soon discovered that almost all such nebulae were receding from Earth. He did not grasp the cosmological implication of this fact, and indeed at the time it was highly controversial whether or not these nebulae were island universe" out side our milky way. Ten year later, Alexander Friedmann, a Russian cosmologist and mathematician, derived the Friedmann equations from Albert Einstein's equation of general relativity, showing that the universe might be expanding in contrast to the static universe model advocated by Einstein. In 1924, Edwin Hubble's measurement of the great distance to the nearest spiral nebulae showed that these system were indeed other galaxies. Independently deriving Friedmann's equation in 1927, Georges Lemaitre , a Belgian Roman Catholic priest ,predicted that the recession of the nebulae was due to the expansion of the universe. In 1931 Lemaitre went further and suggested that the universe began as simple -primeval atom" perhaps echoing previous speculation about the cosmic egg origin of the universe. Cosmology is the attempt to understand in scientific terms the structure and evolution ofthe universe as a whole. This ambition has been with us since the ancient Greeks, even if the developments in modern cosmology have provided a picture of the universe dramatically different from that of Pythagoras, Plato and Aristotle. The cosmological thinking of these figures, e.g. the belief in uniform circular motion of the heavens, was closely related to their philosophical ideas, and it shaped the field of cosmology at least up to the times of Copernicus and Kepler.

\section{Definition}

Tired light is a class of hypothetical redshift mechanisms that was proposed as an alternative explanation for the redshift-distance relationship. ... Z Zwicky himself acknowledged that any sort of scattering of light would blur the images of distant objects more than what is seen. Tired light was an idea that came about due to the observation made by Edwin Hubble that distant galaxies have redshifts proportional to their distance. Redshift is a shift in the spectrum of the emitted electromagnetic radiation from an object toward lower energies and frequencies, associated with the phenomenon of the DopplerEffect. Observers of spiral nebulae such as VestoSlipher observed that these objects now known to be separate galaxies generally exhibited redshift rather than blueshifts independent of where they were located

\section{Methodology}

The way that apparent luminosities could be reduced is through the conversion of photons into particles called axions by intergalactic magnetic fields. There is also a more radical possibility. Ever since the discovery of the cosmological redshift, there has been a nagging doubt about its interpretation as evidence of an expanding universe. Is it possible that the universe is really static, and that photons simply suffer a loss of energy and hence a decrease in frequency as they travel to us, the loss of energy and hence the redshift naturally increasing with the distance that the photons have to travel .

It possible to rule out of all these possibilities by comparing the luminosity distance $d_{L}(\mathrm{z})$ with the angular distance $d_{A}(\mathrm{z})$ of the same distant source .None of the possibilities mentioned above can affect the angular diameter, while the conventional interpretation of redshifts and luminosities provides the model -independent result that $d_{L}(z) / d_{A}(z)$

$=(1+z)^{2}$, so a verification of this ratio can confirm the conventional understanding of $d_{L}(\mathrm{z})$

We can check this formula for $d_{L}(z) / d_{A}(z)$ by a $\rightarrow$ surface

brightness test" . If a light source has absolute luminosity per proper area $\mathrm{L}$, the the apparent luminosity of a patch of area $\mathrm{A}$ will be $\ell=L A / 4 \pi d^{2}$ This patch will subtend a solid angle $\Omega=A / d^{2}{ }_{A}$. The surface brightness $\mathrm{B}$ is defined as the apparent luminosity per solid angle, so

$$
\mathrm{B} \equiv \frac{\ell}{\Omega}=\frac{L d^{2} A}{4 \pi d^{2} L}
$$

In the conventional Big Bang cosmology the ratio $d_{A} / d_{L}$ is given by

$$
\mathrm{B}=(1+Z)^{-4}\left(\frac{L}{4 \pi}\right)
$$

If we can find a class of light source with common value for the absolute luminosity per proper area L,then their surface brightness should be found to be decrease with redshift precisely as $(1+Z)^{-4}$.

\section{Discussion}

The main difference between tired light" theories and the conventional Big Bang theory all rates at the source are decreased by a factor $(1+Z)^{-1}$, while in tired light theory there is no such slowing down. One rate that a slowed down at large redshift in the conventional theory is the rate at which photons are emitted by the sources. This is responsible for one of the two factors of $(1+Z)^{-1}$ for apparent luminosity, the other factor being due to the reduction of energy of individual photons. On the other hand, if the rate of photon emission is not affected by the redshift, then in a static Euclidean universe in which photons lose energy as they travel to us, the apparent luminosity of a distant source $L$ at a distance $d$ will be given by $L / 4 \pi(1+z) d$ 2 , with only a single factor $1+z$ in the denominator to takeaccount of the photon energy loss. That is, the luminosity distance will be $(1+Z)^{1 / 2} \mathrm{~d}$, while the angular diameter distance in a Euclidean universe isjust $d$, so here $d_{L} / d_{A}=(1+z)^{1 / 2}$, and the surface brightness of distant galaxies should decrease as $(1+z)^{-1}$. Lubin and Sandage have used 


\section{International Journal of Science and Research (IJSR) \\ ISSN (Online): 2319-7064 \\ Index Copernicus Value (2015): 78.96 | Impact Factor (2015): 6.391}

the Hubble Space Telescope to compare the surface brightness of galaxies in three distant clusters with redshifts $0.76,0.90$, and 0.92 with the surface brightness measured in closer galaxies. They detect a decrease of $B$ with increasing $z$ that is consistent with Eq. (1.7.2) with reasonable corrections for the effects of galaxy evolution, and is quite inconsistent with the behaviour $B \propto(1+z)^{-1}$ expected in a static universe with tired light."

\section{Conclusion}

In the standard Big Bang cosmology all rates observed from distant source are slowed by factor $1 /(1+Z)$, not just the rate at which photons are emitted .This slowing has been confirmed for the rate of decline of light from some of the type Ia supernovae used by supernova cosmology. The hypothesis that the absolute luminosity is simply correlated with the intrinsic decline time is found to work much better if the observed decline time is taken to be the intrinsic decline time stretched out by factor $1+Z$. Nothing like this would be excepted in static Euclidean universe with redshift attributed to tired light.

\section{References}

[1] F. Zwicky, On the Red Shift of Spectral Lines through Interstellar Space. Proceedings of the National Academy of Sciences 15 (1929): 773-779.[5]

[2] Smeenk, C. (2013). Philosophy of cosmology".In R. Batterman (ed.), Oxford Handbook of Philosophy of Physics.New York, NY: Oxford UniversNity Press, 607652

[3] P. Podsiadlowskiet al., astro-ph/0608324. Evolution may also affect the extinction of light by dust in the host galaxy; see T. Totani and C. Kobayashi, Astrophys J. 526, 65 (1999).

[4] J. Jönssonet al., Astrophys. J. 639, 991 (2006) [astro$\mathrm{ph} / 0506765]$.

[5] S. Weinberg, Rev. Mod. Phys. 61, 1 (1989).

\section{Author Profile}

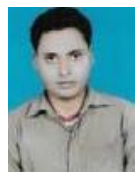

Sumitkumar Bajpeyee, M. Phil \& Research Scholar, BRA Bihar University, Muzaffarpur, Bihar. Worked on Gravitational Radiation: A Stimulating Flux of Energy, volume 5 issue 12 